\title{
Fisher Information inequalities and the Central Limit Theorem
}

\author{
Oliver Johnson \\ Statslab, Cambridge University \\ Andrew Barron \\ Dept of Statistics, Yale University
}

February 1, 2008

\begin{abstract}
We give conditions for an $O(1 / n)$ rate of convergence of Fisher information and relative entropy in the Central Limit Theorem. We use the theory of projections in $L^{2}$ spaces and Poincaré inequalities, to provide a better understanding of the decrease in Fisher Information implied by results of Barron and Brown. We show that if the standardized Fisher Information ever becomes finite then it converges to zero.
\end{abstract}

\section{Introduction}

Bounds on Shannon entropy and Fisher information have long been used in proofs of central limit theorems (CLTs), based on quantification of the change in information as a result of convolution, as in the papers of Linnik (1959),

MSC 2000 subject classification: Primary: 62B10 Secondary: 60F05, 94A17

Key words: Normal Convergence, Entropy, Fisher Information, Poincaré Inequalities, Rates of Convergence

OTJ is a Fellow of Christ's College, Cambridge, who helped support two trips to Yale University during which this paper was written. 
Shimizu (1975), Brown (1982), Barron (1986) and Johnson (2000). Each of these papers have a final step involving completeness or uniform integrability in which a limit is taken without explicitly bounding the information distance from the normal distribution.

The purpose of the present paper is to provide an explicit rate of convergence of information distances, under certain natural conditions on the random variables. Let $X_{1}, X_{2}, \ldots, X_{n}$ be independent identically distributed random variables with mean 0 , variance $\sigma^{2}$ and density function $p(x)$, satisfying Poincare conditions (relating $L^{2}$ norms of mean zero functions to $L^{2}$ norms of the derivative), and let $\phi_{\sigma^{2}}(x)$ be the corresponding $N\left(0, \sigma^{2}\right)$ density. The relative entropy distance is $D(X)=\int p(x) \log \left(p(x) / \phi_{\sigma^{2}}(x)\right) d x$. In the case of random variables with differentiable densities, the Fisher information distance is $J(X)=\sigma^{2} \mathbb{E}\left[\left((d / d x) \log p(X)-(d / d x) \log \phi_{\sigma^{2}}(X)\right)^{2}\right]$ which is related to the Fisher information $I(X)=\mathbb{E}\left[(d / d x \log p(X))^{2}\right]$ via $J(X)=\sigma^{2} I(X)-1$. This is an $L^{2}$ norm between derivatives of log-densities, and gives a natural measure of convergence, stronger than existing theorems, as described in Lemma 1.6. Note that the quantities $D$ and $J$ are scale-invariant, that is $D(a X)=D(X)$ and $J(a X)=J(X)$ for all non-zero $a$.

Let $U_{n}=\left(X_{1}+\ldots+X_{n}\right) / \sqrt{\sigma^{2} n}$ be the standardized sum of the random variables. We show that $D\left(U_{n}\right) \leq 2 R D\left(U_{1}\right) / n \sigma^{2}$ for all random variables with Poincare constant $R$, and that $J\left(U_{n}\right) \leq 2 R J\left(U_{1}\right) / n \sigma^{2}$ for all random variables with density function satisfying a weak differentiability condition.

The present paper builds on ideas in past work which we briefly review here. In examination of the Fisher information a central role is played by the score function $\rho(y)=(d / d y) \log p(y)=p^{\prime}(y) / p(y)$. The score function of the sum of independent random variables can be expressed in terms of the score function of the individual random variables, via a conditional expectation, as has been used in demonstration of convolution inequalities for Fisher information and Shannon entropy (in the work of Stam (1959), Blachman (1965), and others).

In particular, if $Y_{1}$ and $Y_{2}$ are independent and identically distributed with score function $\rho$ then the score $\bar{\rho}(u)$ of the sum $Y_{1}+Y_{2}$ is the projection of $\left(\rho\left(Y_{1}\right)+\rho\left(Y_{2}\right)\right) / 2$ onto the linear space of functions of $Y_{1}+Y_{2}$, so by the Pythagorean identity and rescaling:

$$
\frac{I\left(Y_{1}\right)+I\left(Y_{2}\right)}{2}-I\left(\frac{Y_{1}+Y_{2}}{\sqrt{2}}\right)=2 \mathbb{E}\left(\bar{\rho}\left(Y_{1}+Y_{2}\right)-\frac{\rho\left(Y_{1}\right)+\rho\left(Y_{2}\right)}{2}\right)^{2}
$$


(see Lemma 3.1 for details). Hence, since Equation (11) is positive, one deduces that the Fisher Information is decreasing on the powers-of-two subsequence $S_{k}=U_{2^{k}}$. Equation (II) then quantifies the drop in information $I\left(S_{k}\right)-I\left(S_{k+1}\right)$. Identification of the normal as the limiting distribution arises from examining the difference sequence $I\left(S_{k}\right)-I\left(S_{k+1}\right)$.

Papers by Shimizu (1975), Brown (1982) and Barron (1986) quantify the change in Fisher Information with each doubling of the sample size, deducing convergence to the normal distribution along the powers-of-two subsequence, and convergence of the whole information sequence $I\left(U_{n}\right)$, by subadditvity of $n I\left(U_{n}\right)$. However, these papers only ever consider the behaviour of the Fisher Information for $X \sim Y+Z_{\tau}$ (for $Z_{\tau}$ a small normal perturbation).

However, in general, we can conclude that if the Fisher Information $I\left(S_{k}\right)$ is ever finite, since it is decreasing and bounded below, this difference sequence tends to zero. Thus the interest is in random variables $Y_{1}, Y_{2}$ with score functions for which Equation (II) is small. This expression measures the squared $L^{2}$ difference between a 'ridge function' (a function of the sum $Y_{1}+Y_{2}$ ) and an additive function (a function of the form $g_{1}\left(Y_{1}\right)+g_{2}\left(Y_{2}\right)$ ). From calculus, in general, the only functions $f\left(y_{1}, y_{2}\right)=g_{1}\left(y_{1}\right)+g_{2}\left(y_{2}\right)$ that are both ridge and additive are the linear functions $g_{1}\left(y_{1}\right)=a y_{1}+b, g_{2}\left(y_{2}\right)=$ $a y_{2}+b$, with $a, b_{1}, b_{2}$ constants, that is, the functions for which the derivatives $g_{i}^{\prime}(y)$ are constant and equal.

Previous work, as in Lemma 3.1 of Brown (1982), (see also Barron (1986)) established:

Lemma 1.1 For any functions $f$ and $g$ there exist some $a, b$ such that:

$$
\mathbb{E}\left(g\left(Y_{1}\right)-a Y_{1}-b\right)^{2} \leq \mathbb{E}\left(f\left(Y_{1}+Y_{2}\right)-g\left(Y_{1}\right)-g\left(Y_{2}\right)\right)^{2},
$$

when $Y_{1}, Y_{2}$ are independent identically distributed normals.

Brown takes $g \in L^{2}(\phi)$ and considers the projection $f(s)=\mathbb{E}\left(g\left(Y_{1}\right)+\right.$ $\left.g\left(Y_{2}\right) \mid Y_{1}+Y_{2}=s\right)$. For $Y_{1}, Y_{2}$ normal, the eigenfunctions of this projection are the Hermite polynomials, so he can use expansions in this orthogonal Hermite basis.

The main technique used in the present paper will generalize Lemma 1.1 to a wider class of random variables $Y_{1}, Y_{2}$. For example, consider any $Y_{1}$ and $Y_{2}$ IID with finite Fisher Information $I$. Proposition 2.1 implies that given a 
differentiable ridge function $f\left(y_{1}+y_{2}\right)$, with closest additive function $g$, then for a certain constant $\mu$ :

$$
\mathbb{E}\left(g^{\prime}\left(Y_{1}\right)-\mu\right)^{2} \leq I \mathbb{E}\left(f\left(Y_{1}+Y_{2}\right)-g\left(Y_{1}\right)-g\left(Y_{2}\right)\right)^{2} .
$$

Our (basis-free) proof starts with $f\left(Y_{1}+Y_{2}\right)$, finds its additive part with $g\left(y_{1}\right)=\mathbb{E}_{Y_{2}} f\left(y_{1}+Y_{2}\right)$ and recognises that $g^{\prime}\left(y_{1}\right)=-\mathbb{E}_{Y_{2}} f\left(y_{1}+Y_{2}\right) \rho\left(Y_{2}\right)$. A Cauchy-Schwarz inequality completes the proof as detailed in Section 2 ,

Hence if Equation (11) is small then $\rho$ is close to a function with derivative close to constant in $L^{2}\left(Y_{1}, Y_{2}\right)$. Now Poincaré inequalities provide a relationship between $L^{2}$ norms on functions and the $L^{2}$ norms on derivatives:

Definition 1.2 Given a random variable $Y$, define the Poincaré constant $R_{Y}$ :

$$
R_{Y}=\sup _{g \in H_{1}(Y)} \frac{\mathbb{E} g^{2}(Y)}{\mathbb{E} g^{\prime}(Y)^{2}},
$$

(where $H_{1}(Y)$ is the space of absolutely continuous functions $g$ such that $\operatorname{Var} g(\mathrm{Y})>0, \mathbb{E} g(Y)=0$ and $\left.\mathbb{E} g^{2}(Y)<\infty\right)$, and the restricted Poincaré constant $R_{Y}^{*}$ :

$$
R_{Y}^{*}=\sup _{g \in H_{1}^{*}(Y)} \frac{\mathbb{E} g^{2}(Y)}{\mathbb{E} g^{\prime}(Y)^{2}},
$$

where $H_{1}^{*}(Y)=H_{1}(Y) \cap\left\{g: \mathbb{E} g^{\prime}(Y)=0\right\}$.

For certain $Y, R_{Y}$ is infinite. However, $R_{Y}$ is finite for the normal and other log-concave distributions (see for example Klaasen (1985), Chernoff (1981), Chen (1982), Cacoullos (1982), Borovkov and Utev (1984)). Since we maximise over a smaller set of functions, $R_{Y}^{*} \leq R_{Y}$. Further, for $Z \sim$ $N\left(0, \sigma^{2}\right), R_{Z}^{*}=\sigma^{2} / 2$, with $g(x)=x^{2}-\sigma^{2}$ achieving this (we can show this by expanding $g$ in the Hermite basis).

The other important definition that we shall require is that of weak differentiability, introduced in Fabian and Hannan (1977). Brown and Gajek (1990) and Lehmann and Casella (1998)) discuss this condition, and provide easier to check conditions under which it will hold.

Definition 1.3 A random variable $Y$ has weakly differentiable density $p$ if there exists a function $\rho \in L^{2}(p)$ such that for all $f$ with $\mathbb{E} f(Y+u)^{2}$ finite, the function $g(u)=\mathbb{E} f(Y+u)$ has a derivative $g^{\prime}(u)$ equal to $-\mathbb{E}[f(Y+u) \rho(Y)]$. 
This is a mild technical condition, allowing an exchange of limit and integration. To see the relation to standard differentiability, we can take $f(x)=\mathbb{I}(x \in[a, b])$. Then $g(u)=F(b-u)-F(a-u)$ (where $F$ is the distribution function of $Y$ ), and $g^{\prime}(u)=-\int_{a-u}^{b-u} p(y) \rho(y)$. Thus, for any $a, b$ where the distribution function $F$ is differentiable, $p(b)-p(a)=\int_{a}^{b} p(x) \rho(x) d x$.

Using this, one can extend the Brown inequality Lemma 1.1 to hold (with a constant depending on $I\left(Y_{1}\right)$ and $R_{Y_{1}}$ ) for a wider class of random variables than just normals. Since linear score functions correspond to the family of normal distributions, Equations (11) and (2) provide a means to prove the following Central Limit Theorems:

Theorem 1.4 Given $X_{1}, X_{2}, \ldots$ IID and with finite variance $\sigma^{2}$, define the normalized sum $U_{n}=\left(\sum_{i=1}^{n} X_{i}\right) / \sqrt{n \sigma^{2}}$.

If $X_{i}$ are weakly differentiable with finite restricted Poincaré constant $R^{*}$ then

$$
J\left(U_{n}\right) \leq \frac{2 R^{*}}{n \sigma^{2}} J(X) \text { for all } n
$$

If $X_{i}$ have finite Poincaré constant $R$, then

$$
D\left(U_{n}\right) \leq \frac{2 R}{n \sigma^{2}} D(X) \text { for all } n .
$$

Proof See Sections 2 and 3 for the proof of the Fisher information bound. Notice that for $X$ normal, $2 R^{*}=\sigma^{2}$, so the 'closer to normal $X$ is', the closer the bound becomes to $J(X) / n$.

The relative entropy bound is a corollary. Using an integral form of the de Bruijn identity (Lemma 1 of Barron (1986)), the relative entropy $D(X)$ can be expressed as an integral of $J(\sqrt{1-t} X+\sqrt{t} Z)$ (that is, a linear combination of $X$ and a standard normal $Z$ ). Now, if $X$ has finite Poincaré constant $R$, then for each $t$, the $(\sqrt{1-t} X+\sqrt{t} Z)$ itself has Poincaré constant $\leq R$, so by Theorem 1.4, the result follows.

This $O(1 / n)$ rate of convergence is perhaps to be expected. For example if $X_{i}$ is exponentially distributed, and hence $U_{n}$ has a $\Gamma(n)$ distribution, then $J\left(U_{n}\right)=2 /(n-2)$, consistent with this. In fact, by extending the CramérRao lower bound we deduce that 
Lemma 1.5 If $\mathbb{E} X^{4}$ is finite, then

$$
\liminf _{n \rightarrow \infty} n J\left(U_{n}\right) \geq s^{2} / 3,
$$

where $s$ is the skewness, $m_{3}(X) / m_{2}(X)^{3 / 2}$ (writing $m_{r}(X)$ for the centred rth moment of $X$ ).

Proof For any function $\mathrm{f}$, the positivity of $\mathbb{E}\left(\rho_{U}(U)+f(U)\right)^{2}$ implies that $\mathbb{E} \rho_{U}(U)^{2} \geq \mathbb{E}\left(2 f^{\prime}(U)-f(U)^{2}\right)$, giving a whole family of bounds. Assume that $\mathbb{E} U=0$ and take $f(u)=\left(u-u^{2} m_{3}(U) / m_{4}(U)\right) / m_{2}(U)$. Then $J(U)=$ $m_{2}(U) \mathbb{E} \rho_{U}(U)^{2}-1 \geq m_{3}(U)^{2} /\left(m_{2}(U) m_{4}(U)\right)$. Now, since $m_{2}\left(U_{n}\right)=m_{2}(X)$, $m_{3}\left(U_{n}\right)=m_{3}(X) / \sqrt{n}$ and $m_{4}\left(U_{n}\right)=m_{4}(X) / n+3 m_{2}(X)^{2}(n-1) / n$, the result follows.

Further, this $O(1 / n)$ convergence is consistent with estimates of BerryEsseen type which give a $O(1 / \sqrt{n})$ rate of weak convergence. The following lemma shows the relationship between convergence in Fisher Information, and several weaker forms of convergence:

Lemma 1.6 If $X$ is a random variable with density $f$, and $\phi$ is a standard normal, then:

$$
\begin{aligned}
\sup _{x}|f(x)-\phi(x)| & \leq\left(1+\sqrt{\frac{6}{\pi}}\right) \sqrt{J(X)}, \\
\int|f(x)-\phi(x)| d x \leq 2 d_{H}(f, \phi) & \leq \sqrt{2} \sqrt{J(X)},
\end{aligned}
$$

where $d_{H}(f, \phi)$ is the Hellinger distance $\left(\int|\sqrt{f(x)}-\sqrt{\phi(x)}|^{2} d x\right)^{1 / 2}$.

Proof The first bound comes from Shimizu (1975). The second inequality tightens a bound of Shimizu. Since:

$$
\sqrt{\phi(x)} \frac{\partial}{\partial x} \sqrt{\frac{f(x)}{\phi(x)}}=\frac{1}{2}\left(\frac{f^{\prime}(x)}{\sqrt{f(x)}}+x \sqrt{f(x)}\right),
$$

we deduce from the Poincaré inequality for $\phi$ that:

$$
J(X)=4 \int \phi(x)\left(\frac{\partial}{\partial x} \sqrt{\frac{f(x)}{\phi(x)}}\right)^{2} \geq 4 \int \phi(x)\left(\sqrt{\frac{f(x)}{\phi(x)}}-\mu\right)^{2}=4\left(1-\mu^{2}\right),
$$


where $\mu=\mathbb{E}_{\phi} \sqrt{f / \phi}$, so $d_{H}^{2}(f, \phi)=2(2-2 \mu) \leq 4\left(1-\mu^{2}\right)$.

Recent work by Ball et al (2002) has also considered the rate of convergence of these quantities. Their paper obtains similar results, but by a very different method, involving transportation costs and a variational characterisation of Fisher Information.

Unfortunately, Poincaré constants are not finite for all distributions $Y$. Indeed, as Borovkov and Utev (1984) point out, if $R_{Y}<\infty$, then by considering $g_{n}(x)=|x|^{n}$, we inductively deduce that all the moments of $Y$ are finite. From the Berry-Esseen Theorem we know that only $(2+\delta)$ th moment conditions are enough to ensure an explicit $O\left(1 / n^{\delta / 2}\right)$ rate of weak convergence, for $0<\delta \leq 1$. In Section 0 we describe a proof of Fisher Information convergence under only second moment conditions, though without an explicit rate. This is an extension of Barron's Lemma 2, which only holds for random variables with a normal perturbation.

Theorem 1.7 Given $X_{1}, X_{2}, \ldots$ weakly differentiable IID with finite variance $\sigma^{2}$, define the normalized sum $U_{n}=\left(\sum_{i=1}^{n} X_{i}\right) / \sqrt{n \sigma^{2}}$. If $J\left(U_{m}\right)$ is finite for some $m$ then

$$
\lim _{n \rightarrow \infty} J\left(U_{n}\right)=0 .
$$

Note: This extends Lemma 2 of Barron (1986), which only holds when $X$ is of the form $Y+Z_{\tau}$.

\section{Projection of functions in $L^{2}$}

Although the main application of the following Proposition will concern score functions, we present it as an abstract result concerning projection of functions in $L^{2}\left(Y_{1}, Y_{2}\right)$.

Proposition 2.1 Consider independent random variables $Y_{1}, Y_{2}$ with weakly differentiable densities and functions $f, h_{1}, h_{2}$ such that $\mathbb{E}\left[\left(f\left(Y_{1}+Y_{2}\right)\right)^{2}\right]$ is finite and $\mathbb{E} f\left(Y_{1}+Y_{2}\right)=0$. We find functions $g_{1}, g_{2}$ and a constant $\mu$ such 
that for any $\beta \in[0,1]$ :

$$
\begin{aligned}
\mathbb{E}( & \left.f\left(Y_{1}+Y_{2}\right)-h_{1}\left(Y_{1}\right)-h_{2}\left(Y_{2}\right)\right)^{2} \\
\geq & \mathbb{E}\left(g_{1}\left(Y_{1}\right)-h_{1}\left(Y_{1}\right)\right)^{2}+\mathbb{E}\left(g_{2}\left(Y_{2}\right)-h_{2}\left(Y_{2}\right)\right)^{2} \\
& +(\bar{I})^{-1}\left(\beta \mathbb{E}\left(g_{1}^{\prime}\left(Y_{1}\right)-\mu\right)^{2}+(1-\beta) \mathbb{E}\left(g_{2}^{\prime}\left(Y_{2}\right)-\mu\right)^{2}\right),
\end{aligned}
$$

where $\bar{I}=(1-\beta) I\left(Y_{1}\right)+\beta I\left(Y_{2}\right)$.

Proof Firstly, given $\mathbb{E}\left(f\left(Y_{1}+Y_{2}\right)-h_{1}\left(Y_{1}\right)-h_{2}\left(Y_{2}\right)\right)^{2}$, we can replace $h_{1}$ and $h_{2}$ by functions $g_{1}, g_{2}$ which reduce it even further. This follows since this $L^{2}$ distance is minimised over choices of $h_{1}, h_{2}$ by considering the projections, which remove the additive part:

$$
\begin{aligned}
& g_{1}(u)=\mathbb{E}_{Y_{2}} f\left(u+Y_{2}\right), \\
& g_{2}(v)=\mathbb{E}_{Y_{1}} f\left(Y_{1}+v\right),
\end{aligned}
$$

so the Pythagorean relation tells us that the LHS equals

$\mathbb{E}\left(g_{1}\left(Y_{1}\right)-h_{1}\left(Y_{1}\right)\right)^{2}+\mathbb{E}\left(g_{2}\left(Y_{2}\right)-h_{2}\left(Y_{2}\right)\right)^{2}+\mathbb{E}\left(f\left(Y_{1}+Y_{2}\right)-g_{1}\left(Y_{1}\right)-g_{2}\left(Y_{2}\right)\right)^{2}$.

Having removed the additive part of $f$, we hope that what remains will be small in magnitude. Hence, the inner product of what remains and certain functions of the variables should be small. Specifically we define

$$
\begin{aligned}
& r_{1}(u)=\mathbb{E}_{Y_{2}}\left[\left(f\left(u+Y_{2}\right)-g_{1}(u)-g_{2}\left(Y_{2}\right)\right) \rho_{2}\left(Y_{2}\right)\right], \\
& r_{2}(v)=\mathbb{E}_{Y_{1}}\left[\left(f\left(Y_{1}+v\right)-g_{1}\left(Y_{1}\right)-g_{2}(v)\right) \rho_{1}\left(Y_{1}\right)\right],
\end{aligned}
$$

and show that we can control their norms. Indeed, by Cauchy-Schwarz, for any $u$ :

$$
r_{1}^{2}(u) \leq \mathbb{E}_{Y_{2}}\left(f\left(u+Y_{2}\right)-g_{1}(u)-g_{2}\left(Y_{2}\right)\right)^{2} \mathbb{E} \rho_{2}^{2}\left(Y_{2}\right),
$$

so taking expectations over $Y_{1}$, we deduce that

$$
\mathbb{E} r_{1}^{2}\left(Y_{1}\right) \leq \mathbb{E}\left(f\left(Y_{1}+Y_{2}\right)-g_{1}\left(Y_{1}\right)-g_{2}\left(Y_{2}\right)\right)^{2} I\left(Y_{2}\right) .
$$

Similarly,

$$
\mathbb{E} r_{2}^{2}\left(Y_{2}\right) \leq \mathbb{E}\left(f\left(Y_{1}+Y_{2}\right)-g_{1}\left(Y_{1}\right)-g_{2}\left(Y_{2}\right)\right)^{2} I\left(Y_{1}\right) .
$$

The assumption that $\mathbb{E} f\left(Y_{1}+Y_{2}\right)^{2}$ is finite implies that for almost every $u$ we have $\mathbb{E} f\left(u+Y_{2}\right)^{2}$ finite. Consider any such $u$. The weak differentiability 
of $p_{2}$ (the density of $Y_{2}$ ) gives that the function $g_{1}(u)=\mathbb{E}\left[f\left(u+Y_{2}\right)\right]$ has derivative $g_{1}^{\prime}(u)=-\mathbb{E} f\left(u+Y_{2}\right) \rho_{2}\left(Y_{2}\right)$. Also weak differentiability trivially yields $\mathbb{E} \rho_{2}\left(Y_{2}\right)=0$, so setting $\mu=-\mathbb{E} g_{2}\left(Y_{2}\right) \rho_{2}\left(Y_{2}\right)$, we recognize that $r_{1}(u)$ defined above simplifies to

$$
r_{1}(u)=-\left(g_{1}^{\prime}(u)-\mu\right)
$$

Using the similar expression for $r_{2}(v)=-\left(g_{2}^{\prime}(v)-\mu\right)$, and adding $\beta$ times Equation (3) to $(1-\beta)$ times Equation (44), we deduce the result.

Note: this inequality holds in general, for any weakly differentiable $Y_{1}, Y_{2}$ with finite Fisher Information, whereas previous such expressions have only held in the case of $Y_{i} \sim U_{i}+Z_{\tau}$, for some $U_{i}$.

Note: this inequality allows for independent random variables that are not identically distributed. Armed with it, one may provide Central Limit Theorems giving information convergence to the normal for random variables satisfying a uniform Lindeberg-type condition (see also Johnson (2000)). In certain cases we can provide a rate of convergence.

Note: we can produce a similar expression using a similar method for finitedimensional random vectors $\mathbf{Y}_{1}, \mathbf{Y}_{2}$. Weak differentiability can be defined in this case, and $\rho_{i}=\left(\partial / \partial x_{i}\right)(\log p(x))$ will usually be the $i$ th component of the score vector function $\boldsymbol{\rho}$. Similar analysis in this case can lead to an alternative proof of the Theorems in Johnson and Suhov (2001).

\section{Rate of convergence}

If $Y_{1}, Y_{2}$ have finite restricted Poincaré constants $R_{1}^{*}, R_{2}^{*}$ then we can extend Lemma 1.1 from the case of normal $Y_{1}, Y_{2}$ to more general distributions, providing an explicit exponential rate of convergence of Fisher Information. We can apply Proposition 2.1 because the score functions of sums can be expressed as $L^{2}$ projections.

Lemma 3.1 Let $S=Y_{1}+Y_{2}$ where $Y_{1}$ and $Y_{2}$ are independent and $Y_{2}$ is weakly differentiable with score function $\rho_{2}$. Then $S$ is weakly differentiable with score function

$$
\bar{\rho}(s)=\mathbb{E}\left[\rho_{2}\left(Y_{2}\right) \mid S=s\right] .
$$


Hence for independent weakly differentiable random variables $Y_{1}$ and $Y_{2}$, with score functions $\rho_{1}$ and $\rho_{2}$, writing $\bar{\rho}$ for the score function of $S$

$$
\frac{I\left(Y_{1}\right)+I\left(Y_{2}\right)}{2}-I\left(\frac{Y_{1}+Y_{2}}{\sqrt{2}}\right)=2 \mathbb{E}\left(\bar{\rho}(S)-\frac{\rho_{1}\left(Y_{1}\right)+\rho_{2}\left(Y_{2}\right)}{2}\right)^{2} .
$$

Proof For any square integrable test function $T(u+S)$, define $T_{2}(v)=$ $\mathbb{E}\left[T\left(v+Y_{1}\right)\right]$ so that $T_{2}\left(u+Y_{2}\right)=\mathbb{E}\left[T(u+S) \mid Y_{2}\right]$. Note that $\mathbb{E}\left[\left(T_{2}\left(u+Y_{2}\right)\right)^{2}\right] \leq$ $\mathbb{E}\left[T^{2}(u+S)\right]<\infty$, so that weak differentability can be applied to it. That is we have $\mathbb{E}[T(u+S) \bar{\rho}(S)]=\mathbb{E}\left[T(u+S) \rho_{2}\left(Y_{2}\right)\right]=\mathbb{E}\left[T_{2}\left(u+Y_{2}\right) \rho_{2}\left(Y_{2}\right)\right]=$ $-(d / d u) \mathbb{E} T_{2}\left(u+Y_{2}\right)=-(d / d u) \mathbb{E} T(u+S)$.

Then if both random variables are weakly differentiable, $\bar{\rho}=\mathbb{E}\left[\left(\rho_{1}\left(Y_{1}\right)+\right.\right.$ $\left.\left.\rho_{2}\left(Y_{2}\right)\right) / 2 \mid S=s\right]$. Thus by the Pythagorean identity, the result follows, since we can rescale: $\rho_{a X}(x)=\rho_{X}(x / a) / a$ and $J(a X)=J(X) / a^{2}$.

Proposition 3.2 Consider $Y_{1}, Y_{2}$ IID and weakly differentiable with variance $\sigma^{2}$ and restricted Poincaré constant $R^{*}$. Then

$$
J\left(\frac{Y_{1}+Y_{2}}{\sqrt{2}}\right) \leq J\left(Y_{1}\right)\left(\frac{2 R^{*}}{\sigma^{2}+2 R^{*}}\right) .
$$

Proof Without loss of generality, suppose $Y_{i}$ have mean 0 and variance 1 , since we can just rescale, using $R_{a X}^{*}=a^{2} R_{X}^{*}$. Write $J$ and $I$ for the standardardized and non-standardized Fisher Information of $Y$, and $J^{\prime}$ and $I^{\prime}$ for the corresponding quantities for $\left(Y_{1}+Y_{2}\right) / \sqrt{2}$. By rescaling Lemma 3.1. for projections $g$, writing $\widetilde{\rho}$ for the score function of $\left(Y_{1}+Y_{2}\right) / \sqrt{2}$ :

$$
\begin{aligned}
& J\left(Y_{1}\right)-J\left(\frac{Y_{1}+Y_{2}}{\sqrt{2}}\right) \\
& =\mathbb{E}\left(\tilde{\rho}\left(\frac{Y_{1}+Y_{2}}{\sqrt{2}}\right)-\frac{g\left(Y_{1}\right)+g\left(Y_{2}\right)}{\sqrt{2}}\right)^{2}+\mathbb{E}\left(\rho_{1}\left(Y_{1}\right)-g\left(Y_{1}\right)\right)^{2} .
\end{aligned}
$$

Now, consider the projection of $\widetilde{\rho}$ into the space of additive functions, shown as a plane in Figure团, where $\left(h\left(Y_{1}\right)+h\left(Y_{2}\right)\right) / \sqrt{2}$ is the closest point to $\widetilde{\rho}$ on the line between $-\left(Y_{1}+Y_{2}\right) / \sqrt{2}$ and $\left(\rho\left(Y_{1}\right)+\rho\left(Y_{2}\right)\right) / \sqrt{2}$, so that $\mathbb{E}(g(Y)+Y)^{2} \geq$ $\mathbb{E}(h(Y)+Y)^{2}$.

Further, we know that $h$ corresponds to the value of $\lambda$ which minimises:

$$
\mathbb{E}\left(\tilde{\rho}\left(\frac{Y_{1}+Y_{2}}{\sqrt{2}}\right)-\left(\lambda\left(\frac{\rho\left(Y_{1}\right)+\rho\left(Y_{2}\right)}{\sqrt{2}}\right)+(1-\lambda)\left(\frac{Y_{1}+Y_{2}}{\sqrt{2}}\right)\right)\right)^{2} .
$$




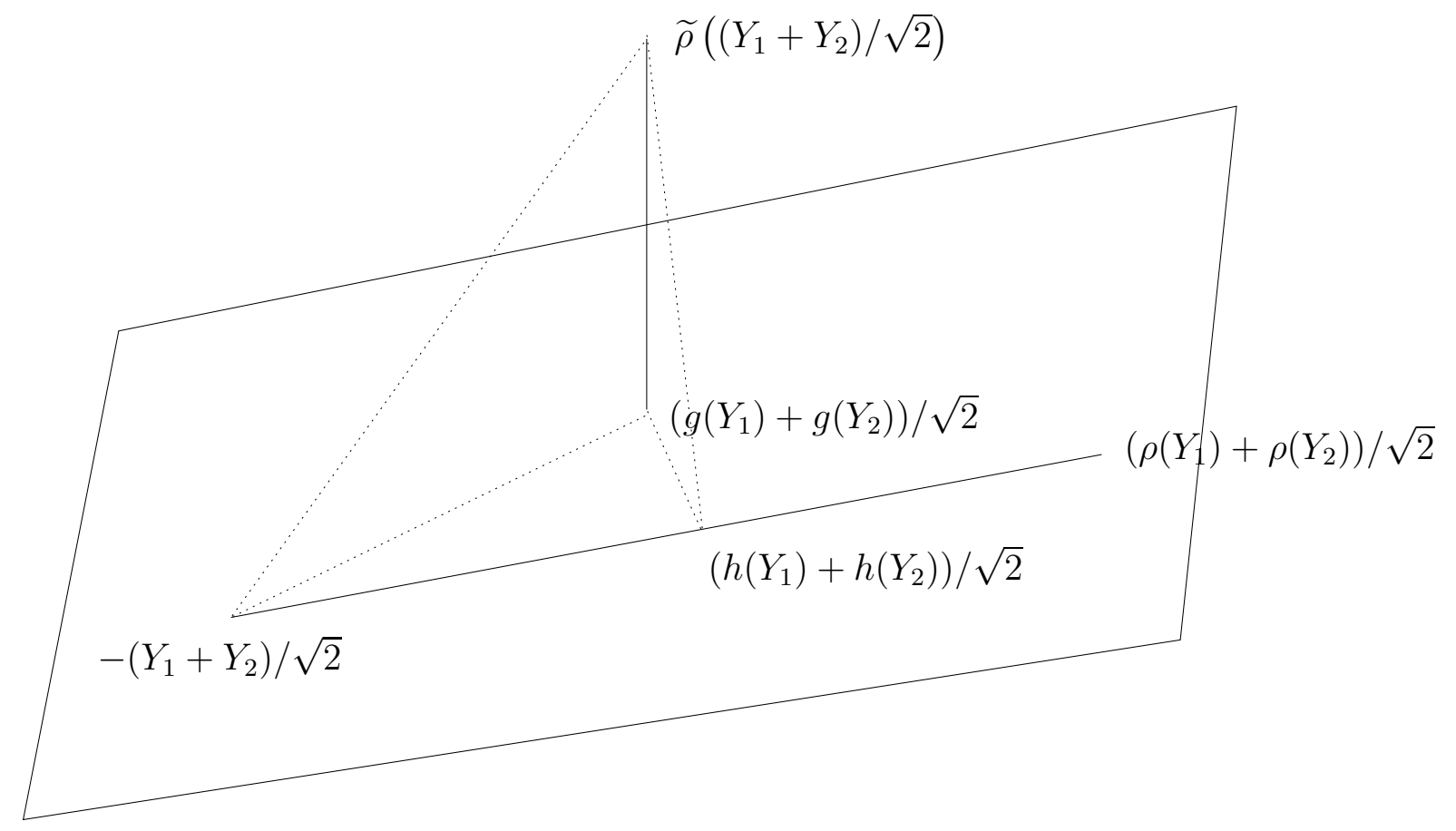

Figure 1: Role of projections

Since in general $\mathbb{E}(U-\lambda V)^{2}$ is minimised at $\lambda=\mathbb{E} U V / \mathbb{E} V^{2}$, in this case the minimising $\lambda=J^{\prime} / J$, so $h$ is $J^{\prime} / J$ of the way along the line. This tells us that $\mathbb{E}(h(Y)+Y)^{2}=\left(J^{\prime} / J\right)^{2} \mathbb{E}(\rho(Y)+Y)^{2}=J^{\prime 2} / J$.

Overall then, we deduce that $\mathbb{E}(g(Y)+Y)^{2} \geq J^{\prime 2} / J$, and by Pythagoras, $\mathbb{E}\left(\widetilde{\rho}\left(\left(Y_{1}+Y_{2}\right) / \sqrt{2}\right)-\left(g\left(Y_{1}\right)+g\left(Y_{2}\right)\right) / \sqrt{2}\right)^{2} \leq J^{\prime}-J^{\prime 2} / J$.

Now applying Proposition 2.1] to the first bracket, we can see that the factor of $I$ in the denominator that Proposition 2.1 implies will actually cancel, simplifying the expression.

$$
\begin{aligned}
J^{\prime}-J^{\prime 2} / J & \geq \mathbb{E}\left(\widetilde{\rho}\left(\frac{Y_{1}+Y_{2}}{\sqrt{2}}\right)-\frac{g\left(Y_{1}\right)+g\left(Y_{2}\right)}{\sqrt{2}}\right)^{2} \\
& \geq \frac{\mathbb{E}\left(g_{1}^{\prime}\left(Y_{1}\right)-\mu\right)^{2}}{2 I} \geq \frac{\mathbb{E}\left(g_{1}\left(Y_{1}\right)-\mu Y_{1}\right)^{2}}{2 R^{*} I} \\
& =\frac{\mathbb{E}\left(g_{1}\left(Y_{1}\right)+Y_{1}\right)^{2}+(-\mu-1)^{2}}{2 R^{*} I} \geq\left(\frac{J^{\prime 2}}{J}+J^{\prime 2}\right) \frac{1}{2 R^{*} I}=\frac{J^{\prime 2}}{2 R^{*} J}
\end{aligned}
$$


since $\mathbb{E} g_{1}^{\prime}\left(Y_{1}\right)-\mu=0$ where $\mu=-I^{\prime}$, and since $\mathbb{E} Y_{1} g_{1}\left(Y_{1}\right)=-1$, so rearranging, we obtain the result.

Note that $g_{1}$ will be absolutely continuous, so we can apply the Poincaré inequality. This follows since $\int_{v}^{w} g_{1}^{\prime}(u) d u=-\int_{v}^{w} \int f(s) p^{\prime}(s-u) d s d u$ and Fubini's Theorem tells us that is $-\int f(s) \int_{v}^{w} p^{\prime}(s-u) d u d s=-\int f(s)(p(s-$ $w)-p(s-v)) d s=g_{1}(w)-g_{1}(v)$, as the random variables have a density everywhere.

A more careful analysis generalises Proposition 3.2, to obtain Theorem 1.4 by performing successive projections onto smaller additive spaces. For a given function $f$, define a series of functions by $f_{n}=f$, and for $m<n$ :

$$
f_{m}\left(\frac{X_{1}+\ldots+X_{m}}{\sqrt{n}}\right)=\mathbb{E}_{X_{m+1}} f_{m+1}\left(\frac{X_{1}+\ldots+X_{m}+X_{m+1}}{\sqrt{n}}\right) .
$$

Further, define $g(u)=\sqrt{n} \mathbb{E} f\left(\frac{X_{1}+\ldots+X_{n-1}+u}{\sqrt{n}}\right)$. At step $i$, we approximate the function $f$ by $f_{i}\left(\left(X_{1}+\ldots+X_{i}\right) / \sqrt{n}\right)$ plus a sum of $g\left(X_{j}\right)$ for $j>i$, which is the best approximation onto the linear space of such partially additive functions.

Lemma 3.3 Defining the squared distance between successive projections to be

$$
t_{i}=\mathbb{E}\left(f_{i}\left(\frac{X_{1}+\ldots+X_{i}}{\sqrt{n}}\right)-f_{i-1}\left(\frac{X_{1}+\ldots+X_{i-1}}{\sqrt{n}}\right)-\frac{1}{\sqrt{n}} g\left(X_{i}\right)\right)^{2},
$$

then for $X_{i}$ IID and weakly differentiable:

$$
t_{i} \geq \frac{(i-1)}{n I(X)} \mathbb{E}\left(g^{\prime}(X)-\mu\right)^{2} .
$$

Proof Evaluate the function

$$
\begin{aligned}
r(z)=\mathbb{E}[ & \left(f_{i}\left(\frac{X_{1}+\ldots+X_{i-1}+z}{\sqrt{n}}\right)-f_{i-1}\left(\frac{X_{1}+\ldots+X_{i-1}}{\sqrt{n}}\right)-\frac{1}{\sqrt{n}} g(z)\right) \\
& \left.\times\left(\rho\left(X_{1}\right)+\ldots \rho\left(X_{i-1}\right)\right)\right],
\end{aligned}
$$


in two different ways. Firstly, again using weak differentiability of $X_{i}$ :

$$
r(z)=-\left(\frac{i-1}{\sqrt{n}}\right)\left(g^{\prime}(z)-\mu\right),
$$

where $\mu=\mathbb{E} f_{i-1}^{\prime}=\mathbb{E} f^{\prime}$. Secondly, we apply Cauchy-Schwarz to $r(z)^{2}$, and take expected values, to deduce that $\mathbb{E} r(X)^{2} \leq t_{i}(i-1) I(X)$. Putting these together, the result follows.

Lemma 3.4 For $X_{i} I I D$, the sum of these squared distances $t_{i}$ is $s_{n}=$ $\sum_{i=1}^{n} t_{i}$, where

$$
s_{m}=\mathbb{E}\left(f_{m}\left(\frac{X_{1}+\ldots+X_{m}}{\sqrt{n}}\right)-\sum_{i=1}^{m} \frac{g\left(X_{i}\right)}{\sqrt{n}}\right)^{2} .
$$

Proof Since $s_{m}=\mathbb{E} f_{m}^{2}-(m / n) \mathbb{E} g^{2}$, and since $t_{m}=\mathbb{E} f_{m}^{2}-\mathbb{E} f_{m-1}^{2}-(1 / n) \mathbb{E} g^{2}$, we can rearrange to obtain:

$$
s_{m}=\left(t_{m}+\mathbb{E} f_{m-1}^{2}+(1 / n) \mathbb{E} g^{2}\right)-(m / n) \mathbb{E} g^{2}=t_{m}+s_{m-1},
$$

so summing the telescoping sum, the result follows.

Combining Lemma 3.3 and Lemma 3.4, we deduce that:

$$
s_{n} \geq \sum_{i=1}^{n} \frac{(i-1)}{n I(X)} \mathbb{E}\left(g^{\prime}(X)-\mu\right)^{2}=\frac{(n-1)}{2 I(X)} \mathbb{E}\left(g^{\prime}(X)-\mu\right)^{2} .
$$

Proof of Theorem 1.4 Again, assuming that $X$ has variance 1, and writing $J^{\prime}$ for $J\left(U_{n}\right)$, and $J$ for $J(X)$, as before we know that $\mathbb{E}(g(X)+X)^{2} \geq J^{\prime 2} / J$ and $s_{n}=\left(\rho_{n}-\sum g\left(X_{i}\right) / \sqrt{n}\right)^{2} \leq J^{\prime}\left(1-J^{\prime} / J\right)$. Hence by Equation (15), we deduce that:

$$
\begin{aligned}
J^{\prime}\left(1-J^{\prime} / J\right) & \geq s_{n} \geq \frac{(n-1)}{2 I(X)} \mathbb{E}\left(g^{\prime}(X)-\mu\right)^{2} \\
& \geq \frac{(n-1)}{2 R^{*} I(X)} \mathbb{E}(g(X)-\mu X)^{2} \\
& \geq \frac{(n-1)}{2 R^{*} I(X)}\left(\frac{J^{\prime 2}}{J}+J^{\prime 2}\right)=\frac{(n-1)}{2 R^{*}} \frac{J^{\prime 2}}{J} .
\end{aligned}
$$


Thus, in general, rescaling gives:

$$
J\left(U_{n}\right) \leq \frac{2 R^{*}}{2 R^{*}+(n-1) \sigma^{2}} J(X),
$$

and the result follows.

Note: for $X$ discrete-valued, $X+Z_{\tau}$ has a finite Poincaré constant, and hence this calculation of an explicit rate of convergence of $J\left(S_{n}+Z_{\tau}\right)$ still holds. Via Lemma 1.6 we know that $S_{n}+Z_{\tau}$ converges weakly for any $\tau$ and hence $S_{n}$ converges weakly to the standard normal.

\section{Convergence of Fisher Information}

We can still obtain convergence of the Fisher Information, though without such an attractive rate of convergence, if the Poincaré constants are not finite. We will need uniform control over the tails of the Fisher Information, and then will bound it on the rest of the region using the projection arguments of Section 2. Recall that for $I(X)$ finite, the density of $X$ is bounded (since $\left.p(y) \leq \int p(x)|\rho(x)| d x \leq \sqrt{I(X)}\right)$.

Definition 4.1 Given a function $\psi$, we define the following class:

$\mathcal{C}_{\psi}=\left\{X: \mathbb{E} X=0, \sigma^{2}=\mathbb{E} X^{2}<\infty, \sigma^{2} \mathbb{E} \rho(X)^{2} \mathbb{I}(|X| \geq \sigma R) \leq \psi(R)\right.$ for all $\left.R.\right\}$

Lemma 4.2 For $X_{1}, X_{2}, \ldots I I D$ with finite variance and finite $I(X)$, then $U_{m} \in \mathcal{C}_{\psi}$ for all $m$ where $\psi(R)=\mathbb{E} \rho(X)^{2} \mathbb{I}(|X| \geq R)+C / R^{1 / 2}$.

Proof We take the common variance to be equal to 1 and use the notation that $p$ and $\rho$ stand for the density and score function of a single $X$, and $p_{r}$ for the density of $X_{1}+\ldots X_{r}$. We know that $U_{m}$ has score function $\rho_{m}(u)=\mathbb{E}\left(\sum_{i} \rho\left(X_{i}\right) \mid U_{m}=u\right) / \sqrt{m}$, so by the conditional version of Jensen's inequality

$$
\rho_{m}(u)^{2} \leq \mathbb{E}\left(\rho\left(X_{1}\right)^{2} \mid U_{m}=u\right)+(m-1) \mathbb{E}\left(\rho\left(X_{1}\right) \rho\left(X_{2}\right) \mid U_{m}=u\right) .
$$


Consider the two terms of Equation (6) separately, firstly writing $W$ for $X_{2}+\ldots X_{m}$ :

$$
\begin{aligned}
& \mathbb{E}_{U_{m}} \mathbb{E}\left[\rho\left(X_{1}\right)^{2} \mid U_{m}\right] \mathbb{I}\left(\left|U_{m}\right| \geq R\right) \\
& \quad \leq \mathbb{E} \rho\left(X_{1}\right)^{2}\left(\mathbb{I}\left(\left|X_{1}\right| \geq R,\left|U_{m}\right| \geq R\right)+\mathbb{I}\left(\left|X_{1}\right|<R,\left|U_{m}\right| \geq R\right)\right) \\
& \quad \leq \mathbb{E} \rho\left(X_{1}\right)^{2}\left(\mathbb{I}\left(\left|X_{1}\right| \geq R\right)+\mathbb{I}(|W| \geq R(\sqrt{m}-1))\right) \\
& \quad \leq \mathbb{E} \rho(X)^{2} \mathbb{I}(|X| \geq R)+\frac{I(X)(m-1)}{R^{2}(\sqrt{m}-1)^{2}}
\end{aligned}
$$

Then for any $u$ :

$$
\begin{aligned}
\mathbb{E} & \left(\rho\left(X_{1}\right) \rho\left(X_{2}\right) \mid U_{m}=u\right) \\
& =\iint \frac{\sqrt{m} p(v) p(w) p_{m-2}(u \sqrt{m}-v-w)}{q_{m}(u)} \rho(v) \rho(w) d v d w \\
& =\frac{\sqrt{m}}{q_{m}(u)} \int p_{m-2}(u \sqrt{m}-x) \int \frac{\partial p}{\partial v}(v) \frac{\partial p}{\partial x}(x-v) d v d x
\end{aligned}
$$

So the second term of Equation (6) is $q_{m}^{\prime}(-R)-q_{m}^{\prime}(R)$ and we need a function $\psi^{\prime}$ such that for all $R$ :

$$
\left|q_{m}^{\prime}(R)\right| \leq \psi^{\prime}(|R|)
$$

For all $m, q_{m}(x) \leq \sqrt{I\left(U_{m}\right)} \leq \sqrt{I}$ for some $I$. Since for any $m$ :

$$
\begin{aligned}
q_{2 m}^{\prime}(u) & =2 \int q_{m}^{\prime}(v) q_{m}(u \sqrt{2}-v) d v \\
& \leq 2^{3 / 4}\left(\int \frac{q_{m}^{\prime}(v)^{2}}{q_{m}(v)} d v\right)^{1 / 2}\left(\int \sqrt{2} q_{m}(v) q_{m}^{2}(u \sqrt{2}-v) d v\right)^{1 / 2} \\
& \leq 2^{3 / 4} \sqrt{I}\left(\sqrt{I} \int \sqrt{2} q_{m}(v) q_{m}(u \sqrt{2}-v) d v\right)^{1 / 2} \\
& \leq(2 I)^{3 / 4} \sqrt{q_{2 m}(u)}
\end{aligned}
$$

(a similar bound will hold for $q_{2 m+1}$ ) and

$$
q_{m}(u) \leq \int_{u}^{\infty}\left|q_{m}^{\prime}(v)\right| d v \leq\left(\int_{u}^{\infty} \frac{q_{m}^{\prime}(v)^{2}}{q_{m}(v)} d v\right)^{1 / 2}\left(\int_{u}^{\infty} q_{m}(v) d v\right)^{1 / 2} \leq \frac{\sqrt{I}}{u}
$$

we deduce that Equation (77) holds, with $\psi^{\prime}(R)=2^{3 / 4} I / R^{1 / 2}$. Note that under a $(2+\delta)$ th moment condition, we obtain $\psi^{\prime}(R)=C / R^{(2+\delta) / 4}$. 
By results of Brown (1982), we know that under a finite variance condition, there exists $\theta(R)$ such that $\mathbb{E} X^{2} \mathbb{I}(|X| \geq \sigma R) \leq \theta(R)$. If in addition, $\mathbb{E}|X|^{2+\delta}$ is finite for some $\delta$, the Rosenthal inequality implies that $\mathbb{E}\left|U_{n}\right|^{2+\delta}$ is uniformly bounded, so we can take $\theta(R)=1 / R^{\delta}$.

The other ingredient we require is a bound on the Poincaré constant $R_{U_{n}}^{T}$ (the Poincaré constant of $U_{n}$ conditioned on $\left|U_{n}\right| \leq T$ ).

Lemma 4.3 If $I(X)$ is finite then there exist $R(T)$ and $N(T)$ such that for all $T, R_{U_{n}}^{T} \leq R(T)$ for $n \geq N(T)$.

Proof Writing $d_{n}=\sup _{A}\left|f_{n}(A)-\phi(A)\right|$ (which tends to zero), since $f_{n}$ is bounded then:

$$
\begin{aligned}
\left|f_{2 n}(x)-\phi(x)\right| \leq & \sqrt{2}\left|\int f_{n}(\sqrt{2} x-y)\left(f_{n}(y)-\phi(y)\right) d y\right| \\
& +\sqrt{2}\left|\int \phi(\sqrt{2} x-y)\left(f_{n}(y)-\phi(y)\right) d y\right| \\
\leq & \sqrt{2}\left(\left\|f_{n}\right\|_{\infty}+\|\phi\|_{\infty}\right) \int\left(f_{n}(y)-\phi(y)\right) I\left(f_{n}(y) \geq \phi(y)\right) d y \\
\leq & (\sqrt{2 I}+\sqrt{1 / \pi}) d_{n}
\end{aligned}
$$

Now, for given $T$, take $N(T)=2 \min \left\{n:(\sqrt{2 I}+\sqrt{1 / \pi}) d_{n} \leq \phi(T) / 2\right\}$. This implies that $f_{n}(x) \geq \phi(T) / 2$, for $x \in[-T, T]$ and $n \geq N(T)$, so $R(T)=$ $2 / \phi(T)$ means

$$
\begin{gathered}
\int_{-T}^{x} y f_{n}(y) d y \leq R(T) f_{n}(x), \text { for } 0 \geq x \geq-T \\
\int_{x}^{T} y f_{n}(y) d y \leq R(T) f_{n}(x), \text { for } 0 \leq x \leq T
\end{gathered}
$$

since the LHS is always less than 1, so by Theorem 1 of Borovkov and Utev (1984) we are done.

Combining these two results gives: 
Proof of Theorem 1.7 Firstly, using the projection inequalities (Proposition 2.1), there exist a function $g$ and constants $\mu, \nu$ such that:

$$
\begin{aligned}
& J\left(U_{n}\right)-J\left(U_{2 n}\right) \\
&=\mathbb{E}\left(\rho_{2 n}\left(U_{2 n}\right)-\frac{1}{\sqrt{2}} \rho_{n}\left(U_{n}\right)-\frac{1}{\sqrt{2}} \rho_{n}\left(U_{n}^{\prime}\right)\right)^{2} \\
& \geq \mathbb{E}\left(\rho_{n}\left(U_{n}\right)-g\left(U_{n}\right)\right)^{2}+\frac{1}{2 I\left(U_{n}\right)} \mathbb{E}\left(g^{\prime}\left(U_{n}\right)-\mu\right)^{2} \\
& \geq \mathbb{E}\left(\rho_{n}\left(U_{n}\right)-g\left(U_{n}\right)\right)^{2} \mathbb{I}\left(\left|U_{n}\right| \leq T\right)+\frac{1}{2 I\left(U_{n}\right)} \mathbb{E}\left(g^{\prime}\left(U_{n}\right)-\mu\right)^{2} \mathbb{I}\left(\left|U_{n}\right| \leq T\right) \\
& \geq \mathbb{E}\left(\rho_{n}\left(U_{n}\right)-g\left(U_{n}\right)\right)^{2} \mathbb{I}\left(\left|U_{n}\right| \leq T\right) \\
&+\frac{1}{2 R_{U_{n}}^{T} I\left(U_{n}\right)} \mathbb{E}\left(g\left(U_{n}\right)-\mu U_{n}-\nu\right)^{2} \mathbb{I}\left(\left|U_{n}\right| \leq T\right) \\
& \geq \frac{1}{1+2 R_{U_{n}}^{T} I\left(U_{n}\right)} \mathbb{E}\left(\rho_{n}\left(U_{n}\right)-\mu U_{n}-\nu\right)^{2} \mathbb{I}\left(\left|U_{n}\right| \leq T\right)
\end{aligned}
$$

Now $\mu=\mathbb{E} \rho_{2 n}^{\prime}\left(U_{n}\right)=-I\left(U_{2 n}\right)$, and $\nu=-\mathbb{E}\left(\rho_{n}+U_{n}\right) \mathbb{I}\left(\left|U_{n}\right| \geq T\right)$, so

$J\left(U_{n}\right) \leq\left(1+2 R_{U_{n}}^{T} I\left(U_{n}\right)\right)\left(J\left(U_{n}\right)-J\left(U_{2 n}\right)\right)+\mathbb{E}\left(\rho_{n}\left(U_{n}\right)-\mu U_{n}-\nu\right)^{2} \mathbb{I}\left(\left|U_{n}\right|>T\right)$,

and hence by Lemmas 4.2 and 4.3, for some function $\zeta(T)$ such that $\zeta(T) \rightarrow 0$ as $T \rightarrow \infty$ :

$$
J\left(U_{n}\right) \leq\left(1+2 R_{U_{n}}^{T} I\left(U_{n}\right)\right)\left(J\left(U_{n}\right)-J\left(U_{2 n}\right)\right)+\zeta(T) .
$$

For any $\epsilon$ we can find $T_{0}$ such that $\zeta\left(T_{0}\right) \leq \epsilon$, for all $n \geq N\left(T_{0}\right)$, then $\left(1+2 R_{U_{n}}^{T} I\left(U_{n}\right)\right)\left(J\left(U_{n}\right)-J\left(U_{2 n}\right) \leq\left(1+2 R\left(T_{0}\right) I\right)\left(J\left(U_{n}\right)-J\left(U_{2 n}\right) \leq \epsilon\right.\right.$ for $n$ sufficiently large.

\section{References}

[1] Ball, K., Barthe, F. and Naor, A. (2002). Entropy jumps in the presence of a spectral gap Preprint.

[2] Barron, A.R. (1986). Entropy and the Central Limit Theorem. Ann. Probab. 14, 336-342. 
[3] Blachman, N.M. (1965) The convolution inequality for entropy powers. IEEE Trans. Inform. Theory 11, 267-271.

[4] Borovkov, A.A. and Utev, S.A. (1984). On an inequality and a related characterisation of the normal distribution. Theory Probab. Appl.28, 219-228.

[5] Brown, L.D. (1982). A proof of the Central Limit Theorem motivated by the Cramér-Rao inequality. In G. Kallianpur, P.R. Krishnaiah, and J.K. Ghosh, editors, Statistics and Probability: Essays in Honour of C.R. Rao, pages 141-148. North-Holland, New York.

[6] Brown, L.D. and Gajek, L. (1990) Information inequalities for the Bayes risk. Ann. Statist.18, 1578-1594.

[7] Cacoullos, Th. (1982). On upper and lower bounds for the variance of a function of a random variable. Ann. Probab. 10, 799-809.

[8] Chen, L.H.Y. (1982) An inequality for the normal distribution. $J$. Multivariate Anal. 12, 306-315.

[9] Chernoff, H. (1981) A note on an inequality involving the normal distribution. Ann. Probab. 9, 533-535.

[10] Fabian, V. and Hannan, J. (1977) On the Cramér-Rao inequality. Ann. Statist. 5, 197-205.

[11] Johnson, O.T. (2000) Entropy inequalities and the Central Limit Theorem. Stochastic Process Appl. 88, 291-304.

[12] Johnson, O.T. and Suhov, Y.M. (2001) Entropy and random vectors. J. Statist Phys. 104, 147-167.

[13] Klaasen, C.A.J. (1985). On an inequality of Chernoff. Ann. Probab. 13, 966-974.

[14] Lehmann, E. and Casella, G. (1998). Theory of point estimation. Springer Texts in Statistics. Springer-Verlag, New York, second edition.

[15] Linnik, Y.V. (1959) An information-theoretic proof of the Central Limit Theorem with the Lindeberg Condition. Theory Probab. Appl. 4, 288299. 
[16] Shimizu, R. (1975) On Fisher's amount of information for location family. In G.P.Patil et al, editor, Statistical Distributions in Scientific Work, Volume 3, pages 305-312. Reidel.

[17] Stam, A.J. (1959) Some inequalities satisfied by the quantities of information of Fisher and Shannon. Inform. and Control 2, 101-112.

\section{Addresses}

O.T.Johnson, Statslab, CMS, Wilberforce Road, Cambridge, CB3 0WB, UK. Email: otj1000@cam.ac.uk.

A.R.Barron, Dept of Statistics, Yale University, PO Box 208290, New Haven, Connecticut 06520-8290, USA. Email: andrew. barron@yale.edu. 